\title{
Foreword by Prof. Dr. Raimund Klinkner, President, German Logistics Association (BVL), Bremen
}

\author{
Raimund Klinkner
}

Published online: 6 February 2009

(C) Springer-Verlag 2009

\section{Dear Reader,}

Logistics is an integral part of practically all economic processes. In the globally networked competitive arena, logistics expertise and performance capability are key success drivers: ever shorter innovation cycles result in a constant stream of new processes. Customers want more specific products and expect these products to be more rapidly available. Modern lifestyles are highly individualistic, and people are ever quicker to change their communication, consumption and working habits. The world is our market; consumers buy and sell online, and we think in terms of working lifetimes and time accounts. Technology continues to advance, and research continues to reveal new interrelationships. Lifelong learning is needed in order to keep up to speed with new developments; at the same time, the half-life of the knowledge acquired by us is decreasing. The global knowledge base doubles in size approximately every 5 years. This means innovation capability and knowledge are two of the key factors in the competitive market.

Knowledge calls for constant awareness and the ability to reflect. Knowledge consists of information placed in the context of experience and deemed to be relevant. People engaged in global value added networks need to possess indepth knowledge as a basis for the development of a regular flow of innovative products and services in order to generate, maintain and extend their competitive edge. Innovation capability in the field of logistics is closely interconnected with research and development in variousabove all interdisciplinary-areas of research: business management and engineering, mathematics and operations

R. Klinkner $(\bowtie)$

German Logistics Association (BVL), Bremen, Germany

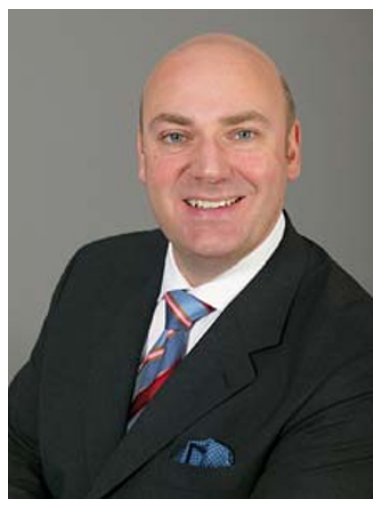

research, information science and intercultural management, to name but a few.

Research findings and hands-on experience together are more than the sum of their parts. Since it was founded 30 years ago the German Logistics Association has devoted its efforts to the exchange of experience and the transfer of knowledge to the real world. Today, the focus is increasingly also on the communication of scientific insights: the new scientific journal "Logistics Research" provides a forum for academic research in the fields of business logistics and logistics engineering. It is a medium for contributions of the highest scientific standard: all submissions are double-blind reviewed by scientific experts. We would be happy to receive papers geared towards the further theoretical development of the fundamental principles or specific aspects of logistics and supply chain management as well as empirical contributions describing the real-world application of the latest knowledge and insights.

This first edition explains how science and research help to find solutions to complex problems in a dynamic environment. The core theme of "Hypercompetition" profiles approaches that allow a fast and flexible network-based response to new technologies, shorter innovation cycles and the increasing pressure of international competition.

I hope this journal makes for an "interesting read" and that it supplies new ideas and fresh stimuli.

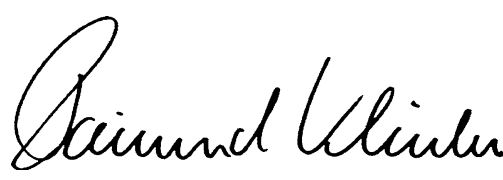

